\title{
Ordered structure and twin boundary of triblock copolymer/silica mesophase thin films
}

\author{
Chi Won Ahn and Theo Rasing a) \\ NSRIM, University of Nijmegen, Toernooiveld 1, 6525 ED, Nijmegen, The Netherlands \\ Mahn Won Kim \\ Department of Physics, Korea Advanced Institute of Science and Technology (KAIST), Daejon 305-701, \\ Republic of Korea
}

(Received 25 April 2003; accepted 27 August 2003)

\begin{abstract}
We have observed a twin boundary in a triblock copolymer/silica mesophase thin film, produced by spinning an acidic water-ethanol-tetraethoxysilane solution of a triblock copolymer on a hydrophilic treated silicon substrate. The highly ordered structure of the mesophase thin film is formed by ordering of self-assembled triblock copolymer micelles and was characterized with x-ray reflectivity and transmission electron microscopy. The mesophase thin film consists of a face-centered-cubic lattice and the orientation of the film surface is the $\{111\}$ plane. Furthermore, a $\{111\}$ twin plane is observed in the mesophase thin film. (C) 2003 American Institute of Physics.
\end{abstract}

[DOI: $10.1063 / 1.1622433$ ]

In the last ten years, surfactant/silica mesophase materials have been synthesized in a variety of ordered forms showing lamellar, hexagonal, and cubic phases. ${ }^{1-3}$ These are called a new kind of crystal because they have long-range order like crystalline solids. ${ }^{4,5}$ Real crystalline solids have imperfections or defects, such as point defects (vacancies and interstitials), line defects (dislocations), and planar defects (twins, stacking faults, and grain boundaries). ${ }^{6}$ In particular, the latter have an orientation difference in the atomic arrangement across the interface. In polycrystalline materials, planar defects (interfaces) are always present, and their control is very important to improve the physical properties of materials. $^{7}$

For mesophase materials, one of the main objectives is to improve the degree of ordering. Mesophase crystals also have defects, but these have rarely been studied except for the topological defect of a hexagonal mesophase material. ${ }^{8,9}$ By using an electron microscope, Feng et al. observed pairs of line defects (dislocations) as a form of dislocation dipole and disclination quadrapole in a surfactant/silica mesophase powder. The pairing of dislocations minimizes the strain energy of the deformation and forms a curved surface. ${ }^{8}$ Hillhouse et al. also found topological defects in mesophase films consisting of rounded granules, dislocations, and disclinations. ${ }^{9}$ In the case of a mesophase thin film, the formation and growth of mesophase structures is restricted within the film thickness. Therefore mesophase thin films can form planar defects, as has been reported for block copolymer thin films. ${ }^{10,11}$

In this study, we synthesized thin films of cubic mesophase silica by using triblock copolymers that form a cubic phase in high concentrated solution. The ordered structure of triblock copolymer/silica mesophase thin films was characterized by $\mathrm{x}$-ray reflectivity and transmission electron mi-

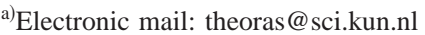

croscopy. We have observed a twin defect and will discuss the origin of its formation.

Poly(ethylene oxide)-poly(propylene oxide)-poly(ethylene oxide $)\left(\mathrm{EO}_{M}-\mathrm{PO}_{N}-\mathrm{EO}_{M}, \quad M\right.$ and $N$ are the number of monomers, $\mathrm{F} 108=\mathrm{EO}_{133}-\mathrm{PO}_{50}-\mathrm{EO}_{133}, \quad \mathrm{~F} 68$ $\left.=\mathrm{EO}_{77}-\mathrm{PO}_{29}-\mathrm{EO}_{77}\right)$ triblock copolymers, were obtained from Poloxamer Co. Tetraethoxysilane [TEOS, $\mathrm{Si}\left(\mathrm{C}_{2} \mathrm{H}_{5} \mathrm{O}\right)_{4}$ ] with $>98 \%$ purity was obtained from Acros Co. Ethanol (Merck, 99.9\%, ACS) and double distilled water were used. Hydrochloric acid $(\mathrm{HCl}, 35 \mathrm{~g} / \mathrm{ml})$ was obtained from Aldrich Chemical. A mixture (70:30 v/v) of sulfuric acid $\left(\mathrm{H}_{2} \mathrm{SO}_{4}, 95\right.$ $\mathrm{g} / \mathrm{ml})$ and hydrogen peroxide $\left(\mathrm{H}_{2} \mathrm{O}_{2}, 35 \mathrm{~g} / \mathrm{ml}\right)$ was used to clean the silicon wafer (Mitsubishi Material Silicon Co.) for 30 min at $90{ }^{\circ} \mathrm{C} .{ }^{12}$ All chemicals were used as received. The cleaned silicon surface becomes hydrophilic. The cleaned substrates were immediately rinsed and kept in distilled water.

To obtain a typical reaction solution, $0.008 \mathrm{M}$ of triblock copolymer was dissolved by stirring a solution of $4.7 \mathrm{ml}$ water $(p \mathrm{H}=1.4$, mixed with $\mathrm{HCl}$ solution) and $22 \mathrm{ml}$ ethanol; then $6.4 \mathrm{ml}$ of TEOS was added and stirred at room temperature. ${ }^{13}$ The mesophase thin film was prepared by spin-coating with a spinning rate of $5000 \mathrm{rpm}$ and a spinning time of $30 \mathrm{~s}$. After spin-coating, the samples were dried at room temperature.

For the characterization of the synthesized thin film, we used transmission electron microscopy (TEM, CM20, Philips, at $120 \mathrm{keV}$ ), x-ray reflectometry (beamline 3C2 of the Pohang Light Source, Korea, x-ray wavelength $=1.5402 \AA$ ), and atomic force microscopy (AFM, AutoProbe CP20, PSI). TEM samples (for plane view) of the thin films were prepared by taking them off the substrate and putting them on a carbon-coated $\mathrm{Cu}$ grid. Ultramicrotomy (Ultracut UCT, Leica) was used to make cross-sectioned TEM samples with a thickness of about $70 \mathrm{~nm}$. Dynamic light scattering (DLS, BI-200SM, Brookhaven Instruments) was used to measure the micelle size of the triblock copolymer in solution. 


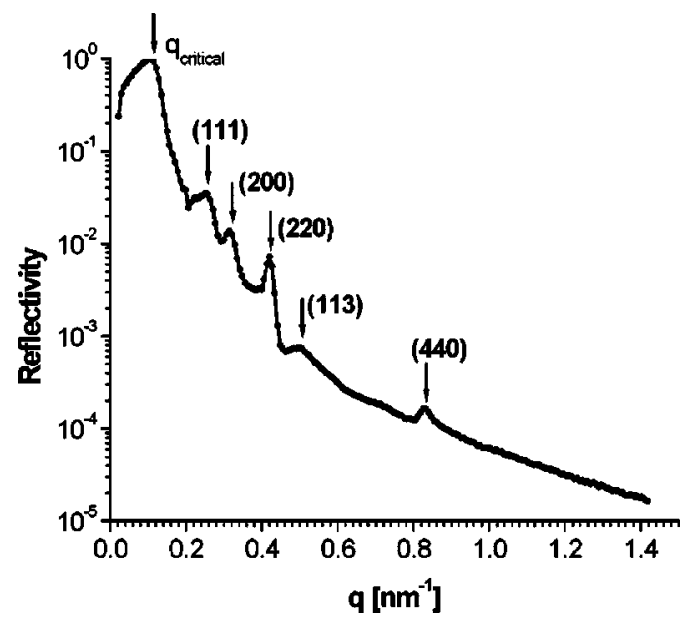

FIG. 1. The measured $x$-ray reflectivity from the F108/silica mesophase film. Indexed diffraction peaks correspond to Bragg reflection planes in the fcc system. $q_{\text {critical }}$ is the critical angle for the total reflection of the incident $\mathrm{x}$ ray.

The F108 and F68 triblock copolymers are amphiphilic. ${ }^{14}$ They act as a structure-directing agent in an acidic water-ethanol-TEOS solution by self-assembly. In our previous study, ${ }^{13}$ the higher monomer number ratio of EO to $\mathrm{PO}$ of $\mathrm{EO}_{M}-\mathrm{PO}_{N}-\mathrm{EO}_{M}$ triblock copolymers was shown to increase the structural ordering of mesophase thin films. Therefore, we could control the structural ordering of the mesophase thin films by increasing the EO to PO monomer number ratio to make a cubic structure. ${ }^{3,13}$ In the case of F108 and F68 triblock copolymers, the EO to PO monomer ratio has the same highest value of 5.3.

The structure of the synthesized F108/silica mesophase thin film was analyzed by $x$-ray reflectivity. Figure 1 gives the $\mathrm{x}$-ray reflectivity data and plane indices of the F108/silica mesophase thin film, that are consistent with a face-centered-

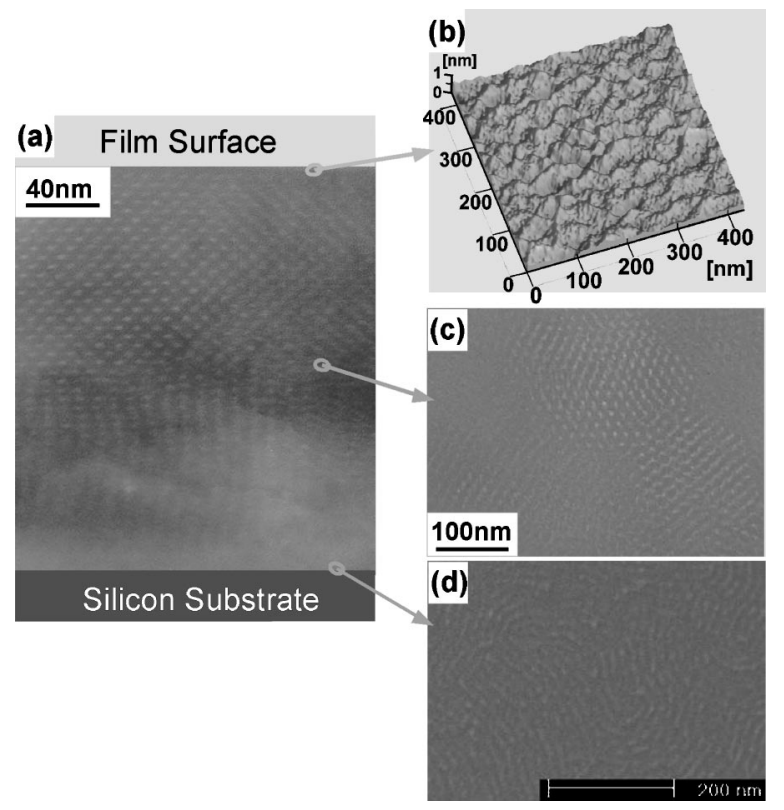

FIG. 2. Three-dimensional view of the microstructure of the F108/silica mesophase thin film; (a) TEM image of the cross-sectioned film, (b) AFM image of the film surface, (c) TEM image of the normal direction of the film, and (d) SEM image at the film/substrate interface after detaching the film from the substrate.
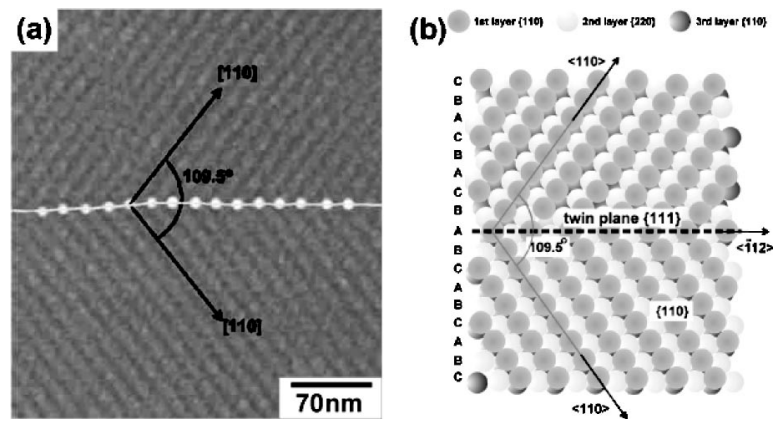

FIG. 3. (a) Plane view of mesophase F108/silica thin film by TEM. White spots and white line denote a $\{111\}$ twin plane. (b) Schematic structure of fcc crystal system and a $\{111\}$ twin plane. The twin boundary is projected on the $\{110\}$ plane along the $\langle\overline{1} 12\rangle$ axis. The letters (A, B, and $\mathrm{C})$ on the left side denote the packing sequence of the $\{111\}$ planes.

cubic (fcc) lattice structure. Using Bragg's law and a least squares method, the lattice parameter of the F108/silica mesophase thin film was found to be $21 \mathrm{~nm} .{ }^{15}$ Figure 2 gives a three-dimensional view of the internal and external microstructure of the F108/silica mesophase thin film. The TEM picture of a cross section [Fig. 2(a)] shows that the ordering is most perfect near the film surface. Figure 2(b) is an AFM image of the film surface, showing that the surface morphology has a nanometer scale roughness. Figure 2(c) is a TEM plane view of the mesophase thin film showing some grains and grain boundaries. Figure 2(d) is a scanning electron microscope (SEM) image of the thin film/substrate interface after detaching the thin film from the hydrophilic silicon substrate. It shows that the thin film/substrate interface has a less ordered structure than the upper side of the mesophase thin film.

Among the plane-view TEM images of the F108/silica mesophase thin film, we can discover a $\{111\}$ twin plane along the $\langle\overline{1} 12\rangle$ axis of the fcc lattice system [Fig. 3]. According to the X-ray diffraction results [Fig. 4] and the TEM images [Figs. 2(a) and 5(a)], the F68/silica mesophase thin film is more ordered than the F108/silica one. The peaks in Fig. 4 are the diffraction of the (111) plane because the thin film aligned parallel to the (111) plane direction. By decreasing the molecular weight from $14600 \mathrm{~g} / \mathrm{mol}$ (F108) to 8400 $\mathrm{g} / \mathrm{mol}$ (F68), the triblock copolymers formed smaller micelles and the diffraction peak of the synthesized mesophase thin film became sharper. The position $2 \theta$ of the (111) dif-

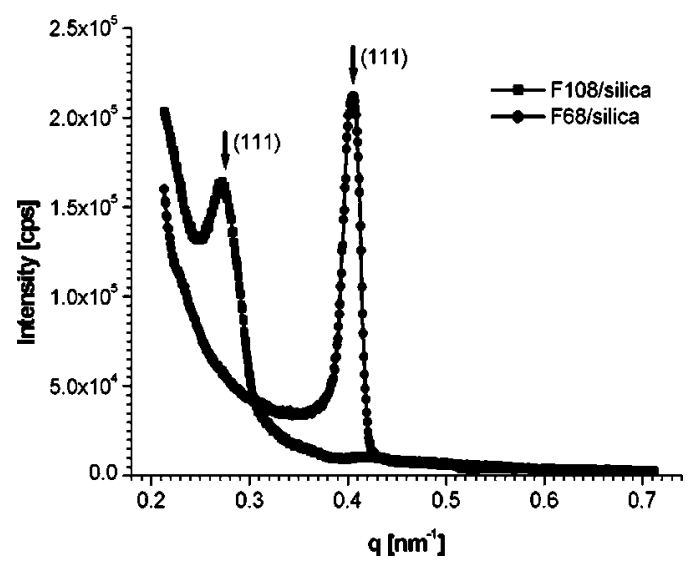

FIG. 4. X-ray diffraction peaks of the (111) plane of F108/silica and F68/

silica mesophase thin films. 

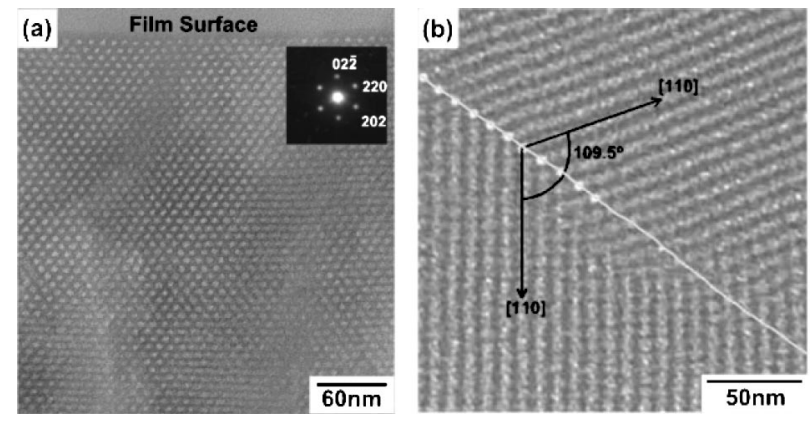

FIG. 5. (a) Cross-sectioned TEM lattice image and SADP in a [111] direction (zone axis). (b) TEM image of the twin boundary in a F68 triblock copolymer/silica mesophase thin film. White spots and solid line denote the $\{111\}$ twin plane.

fraction peak is shifted from $0.76^{\circ}$ to $1.14^{\circ}$, yielding an interplanar spacing $\left(d_{111}\right)$ of 11.5 and $7.7 \mathrm{~nm}$, respectively. From the DLS experiments, we obtained the F108 micelle size $(11 \pm 1.5 \mathrm{~nm})$ and the F68 micelle size $(6.5 \pm 1 \mathrm{~nm})$.

Figure 5(a) is a cross-sectional TEM lattice image of the F68/silica mesophase thin film in the [111] zone axis. Selected area diffraction patterns (SADP) denote that the mesophase thin film displays a shrinkage in the perpendicular direction nearby the surface. From this TEM image, the internal structure of the F68/silica thin film appears to be a highly ordered fcc structure. In the TEM image of the plane view [Fig. 5(b)], we also observed a twin boundary in the F68/silica mesophase thin film. There are two possible mechanisms that may explain why the triblock copolymer/ silica mesophase thin film has a twin boundary. One is that a growth twin can arise during the growth of the fcc mesophase domains from the micelle solution. During this growth process of the domains in the thin film, there is a growth limit on the domains along the vertical direction to the substrate because a thin film is a confined system (the film thickness is below $300 \mathrm{~nm}$ ). Therefore, the domains grow along a direction parallel to the substrate. If during this process two domains meet, they make an interface, and the energy of that interface can be minimized by the formation of a twin, as a twin boundary is one of the lowest energy interfaces. The other possible mechanism would be a mechanical twin by shear energy during spin coating, but the spinning rate (shear force) has to be more than $8000 \mathrm{rpm}$ to form a twin boundary. ${ }^{16,17}$ Therefore, the twin boundaries observed in our experiment are mainly growth twins because the shear force of our experiment is too low for the formation of mechanical twins.

In this work, we synthesized highly ordered fcc mesophase thin films from a acidic water-ethanol-TEOS solution of F108 and F68 micelles. The molecule sizes of the triblock copolymers strongly effect the micelle ordering. F68 made smaller micelles than F108 and formed a more ordered mesophase structure. We have shown a twin boundary in F108/silica and F68/silica mesophase thin films. It is a growth twin formed to reduce the interface energy during the growth of the domains in the fcc mesophase thin films.

We thank Dr. Y. G. Kim (KRISB, Korea) and Dr. G. S. Park (SAIT, Korea) for helpful analysis with TEM. X-ray experiments at PLS were supported in part by MOST and POSCO. This work was partially supported by the Dutch Technology Foundation (STW) and the Advanced Backbone IT Technology Development Project (IMT-200-B3-2), Ministry of Information and Communication.

${ }^{1}$ C. T. Kresge, M. E. Leonowicz, W. J. Roth, J. C. Vartuli, and J. S. Beck, Nature (London) 359, 710 (1992).

${ }^{2}$ G. D. Stucky, Q. Huo, A. Firouzi, and B. F. Chmelka, Progress in Zeolite and Microporous Materials 105, 3 (1997).

${ }^{3}$ D. Zhao, Q. Huo, F. Feng, B. F. Chmelka, and G. D. Stucky, Acta Mater. 120, 6024 (1998).

${ }^{4}$ N. A. Melosh, P. Davidson, and B. F. Chmelka, J. Am. Chem. Soc. 122, 823 (2000).

${ }^{5}$ Y. Sakamoto, M. Kaneda, O. Terasaki, D. Y. Zhao, J. M. Kim, G. Stucky, H. J. Shin, and R. Ryoo, Nature (London) 408, 449 (2000).

${ }^{6}$ G. A. Chadwick and D. A. Smith, Grain Boundary Structure and Properties (Academic, London, 1976), Chaps. 12, 13.

${ }^{7}$ J. M. Howe, Interface in Materials (Wiley, New York, 1997).

${ }^{8}$ J. Feng, Q. Huo, P. M. Petroff, and G. D. Stucky, Appl. Phys. Lett. 71, 1887 (1997)

${ }^{9}$ H. W. Hillhouse, J. W. van Egmond, and M. Tsapatsis, Chem. Mater. 12, 2888 (2000)

${ }^{10}$ S. P. Gido and E. L. Thomas, Macromolecules 27, 6137 (1994).

${ }^{11}$ H. J. Dai, N. P. Balsara, B. A. Garetz, and M. C. Newstein, Phys. Rev. Lett. 77, 3677 (1996).

${ }^{12}$ S. R. Wasserman, Y. T. Tao, and G. M. Whitesides, Langmuir 5, 1074 (1989).

${ }^{13}$ C. W. Ahn, J. K. Park, and M. W. Kim, Mol. Cryst. Liq. Cryst. 37, 477 (2001).

${ }^{14}$ P. Alexandridis and T. A. Hatton, Colloids Surf., A 96, 1 (1996).

${ }^{15}$ B. D. Cullity, Elements of X-ray Diffraction, 2nd ed. (Addison-Wesley, Reading, MA, 1978).

${ }^{16}$ E. Eiser, F. Molino, G. Porte, and X. Pithon, Rheol. Acta 39, 201 (2000).

${ }^{17}$ I. W. Hamley, Curr. Opin. Colloid Interface Sci. 5, 342 (2000). 